\title{
Percutaneous left atrial appendage occlusion in South Africa
}

Atrial fibrillation (AF) is a global challenge, with an estimated prevalence of $1-2 \%$ in the general population. ${ }^{[1]}$ The prevalence increases with age, ${ }^{[1]}$ and AF affects up to $15 \%$ of octogenarians. AF is independently associated with mortality, cardiac failure and non-fatal stroke. $^{[1,2]}$ Thromboprophylaxis for high-risk patients is provided with oral anticoagulation (OAC) using vitamin $\mathrm{K}$ antagonists (VKAs), such as warfarin. Meta-analysis of 5 randomised clinical trials demonstrated that $\mathrm{OAC}$ results in a relative risk reduction of $68 \%$ for ischaemic stroke. ${ }^{[3]}$ Over the past decade, the use of novel oral anticoagulants (NOACs) has gained traction, with randomised trials showing non-inferiority of these drugs for the prevention of ischaemic stroke compared with VKAs, as well as a lower risk of cerebrovascular haemorrhage. VKAs or NOACs, however, are best avoided in patients who are intolerant to their effects (e.g. lifethreatening haemorrhage), non-adherent or have an unacceptably high bleeding risk.

Percutaneous left atrial appendage occlusion (LAAO) is an alternative approach to thromboprophylaxis in AF, predicated on the fact that $<10 \%$ of clinical emboli in non-valvular AF originate outside the LAA. ${ }^{[4]}$ The LAA is excluded as an embolic source by placement of an occluder device into the ostium of the LAA via the femoral vein and interatrial, transseptal puncture.

The Watchman Left Atrial Appendage System for Embolic Protection in Patients With Atrial Fibrillation (PROTECT AF) ${ }^{[5]}$ trial prospectively randomised 707 patients with non-valvular AF to LAAO or warfarin. After 2 years of follow-up, the cumulative adverse event rate (stroke, cardiovascular/unexplained death or systemic embolism) was 5.9\% for the LAAO arm, compared with 8.3\% for the warfarin group, indicating non-inferiority. Although the Prospective Randomized Evaluation of the LAA Closure Device in Patients With Atrial Fibrillation Versus Long-Term Warfarin Therapy (PREVAIL) $^{[6]}$ trial (mimicking the original PROTECT AF protocol) failed to demonstrate non-inferiority of LAAO compared with warfarin, a significantly lower adverse event rate was recorded for LAAO ${ }^{[6,7]}$ More recently, the Left Atrial Appendage Closure vs. Novel Anticoagulation Agents in Atrial Fibrillation (PRAGUE-17) study ${ }^{[8]}$ performed a head-to-head comparison between NOACs and LAAO, showing non-inferiority $(p=0.004) \cdot{ }^{[9]}$ Procedural success rates are high and complication rates low with modern devices: the Amulet LAAO device (Abbot Vascular, St Paul, MN, USA) has demonstrated $99 \%$ implantation success, ${ }^{[10,11]}$ with only a $0.2 \%$ stroke and $0.9 \%$ major vascular complication rate. In a large, pooled study of 5-year PROTECT AF and PREVAIL trial data performed in 2019, LAAO was not only cost-effective, but even demonstrated a cost advantage compared with warfarin and NOACs. ${ }^{[7]}$

While VKAs remain the mainstay of thromboprophylaxis in South Africa (SA), many AF patients are receiving suboptimal protection with OAC due to non-adherence, contraindications and complications. ${ }^{[12]}$ In the Atrial fibrillation Clopidogrel Trial with Irbesartan for prevention of Vascular Events (ACTIVE W), SA participants demonstrated international normalised ratios (INRs) in the therapeutic range only $40 \%$ of the time. ${ }^{[13]}$ Clarkesmith et al. ${ }^{[14]}$ performed a systematic review in 2017 regarding interventions for OAC compliance in $\mathrm{AF}$ and found that there is insufficient evidence to draw definitive conclusions regarding the impact of educational or behavioural interventions. Very limited local data exist on percutaneous LAAO, with a single case series published in 2013. ${ }^{[15]}$ This procedure, however, is an attractive alternative to OAC, but is currently performed only in a few centres in SA. LAAO should be considered by referring clinicians as an alternative to $\mathrm{OAC}$ in patients who are intolerant, non-adherent or who have a high bleeding risk.

\section{André P Dippenaar}

Specialist Physician, Netcare Kuils River Hospital, Cape Town, South Africa dradippenaar@gmail.com

\section{Pieter van der Bijl}

Cardiologist, Netcare Kuils River Hospital, Cape Town, South Africa

\section{Marshall J Heradien}

Cardiologist, Department of Medicine, Faculty of Medicine and Health Sciences, Stellenbosch University, Cape Town, and Netcare Kuils River Hospital, Cape Town, South Africa

\section{André Saaiman}

Cardiologist, Netcare Kuils River Hospital, Cape Town, South Africa

1. Kirchhof P, Benussi S, Kotecha D, et al. 2016 ESC guidelines for the management of atrial fibrillation developed in collaboration with EACTS. Eur Heart J 2016;37(38):2893-2962. https://doi.org/10.1093/ eurhearti/ehw210

2. Correction to: $2019 \mathrm{AHA} / \mathrm{ACC} / \mathrm{HRS}$ focused update of the $2014 \mathrm{AHA} / \mathrm{ACC} / \mathrm{HRS}$ guideline for the management of patients with atrial fibrillation: A Report of the American College of Cardiology/ American Heart Association Task Force on Clinical Practice Guidelines and the Heart Rhythm Society. Circulation 2019;140(6):e285. https://doi.org/10.1161/cir.0000000000000719

3. Risk factors for stroke and efficacy of antithrombotic therapy in atrial fibrillation. Arch Intern Med 1994;154(13):1449-1457. https://doi.org/10.1001/archinte.1994.00420130036007

4. Sievert $\mathrm{H}$. Lesh MD Trepels T et al. Percutaneous left atrial appendage transeatheter occlusion to Severt $\mathrm{H}$ Lesh MD, Trepels $\mathrm{T}$, et al. Percutaneous left atrial appendage transcatheter occlusion prevent stroke in high-risk patients with atrial fibrillation. Circulation 2002;105(16):1887-1889.

5. Reddy VY, Doshi SK, Sievert H, et al. Percutaneous left atrial appendage closure for stroke prophylaxis in patients with atrial fibrillation: 2.3-year follow-up of the PROTECT AF (Watchman Left Atria Appendage System for Embolic Protection in Patients With Atrial Fibrillation) trial. Circulation 2013;127(6):720-729. https://doi.org/10.1161/circulationaha.112.11438

6. Holmes DR, Jr, Kar S, Price MJ, et al. Prospective Randomized Evaluation of the Watchman Left Atrial Appendage Closure Device in Patients With Atrial Fibrillation Versus Long-Term Warfarin Therapy: The PREVAIL trial. J Am Coll Cardiol 2014;64(1):1-12. https://doi.org/10.1016/j.jacc.2014.04.029

7. Reddy VY, Doshi SK, Kar S, et al. 5-year outcomes after left atrial appendage closure: From the PREVAIL and PROTECT AF trials. J Am Coll Cardiol 2017;70(24):2964-2975. https://doi. org/10.1016/j.jacc.2017.10.021

8. Osmancik P, Tousek P, Herman D, et al. Interventional Left Atrial Appendage Closure vs. Novel Anticoagulation Agents in Atrial Fibrillation indicated for long-term anticoagulation (PRAGUE-17 study). Am Heart J 2017;183:108-114. https://doi.org/10.1016/j.ahj.2016.10.003

9. Oty. Am Heart J 2017,183.108-114. hltps.//doi.org/10.1016/).ahj.2016.10.003 Osmancik P, Herman D, Neuzil P, et al. Left atrial appendage closure versus direct oral anticoagulants
in high-risk patients with atrial fibrillation. J Am Coll Cardiol 2020;75(25):3122-3135. https://doi.

10. Lakkireddy D, Windecker S, Thaler D, et al. Rationale and design for AMPLATZER amulet left atrial Lakkireddy D, Windecker S, Thaler D, et al. Rationale and design for AMPLATZER amulet left atria
appendage occluder IDE randomized controlled trial (Amulet IDE trial). Am Heart J 2019;211:45-53.

appendage occluder IDE randomized con
https://doi.org/10.1016/j.ahj.2018.12.010 1. Hildick-Smith D, Landmesser U, Camm AJ, et al. Left atrial appendage occlusion with the Amplatzer" Amulet" device: Full results of the prospective global observational study. Eur Heart J 2020;41(30):2894-2901. https://doi.org/10.1093/eurhearti/ehaa169

12. Hylek EM, Evans-Molina C, Shea C, Henault LE, Regan S. Major hemorrhage and tolerability of warfarin in the first year of therapy among elderly patients with atrial fibrillation. Circulation 2007;115(21):2689-2696. https://doi.org/10.1161/circulationaha.106.653048

3. Aalbers J. South Africảs poor warfarin control raises questions of benefit above other anticoagulant therapies in atrial fibrillation. Cardiovasc J Afr 2011;22(4):220.

4. Clarkesmith DE, Pattison HM, Khaing PH, Lane DA. Educational and behavioural interventions for anticoagulant therapy in patients with atrial fibrillation. Cochrane Database Syst Rev for anticoagulant therapy in patients with atrial fibrillation. Cochrane Databat

15. Abelson M. Left atrial appendage closure in patients with atrial fibrillation in whom warfarin is contra-indicated: Initial South African experience. Cardiovasc J Afr 2013;24(4):107-109. https://doi. org/10.5830/CVJA-2013-018

S Afr Med J 2021;111(7):609. https://doi.org/10.7196/SAMJ.2021.v111i7.15536 